This is an Open Access article, distributed under the terms of the Creative Commons Attribution licence (http://creativecommons.org/licenses/by/4.0/), which permits unrestricted re-use, distribution, and reproduction in any medium, provided the original work is properly cited.

doi: $10.1017 /$ jfm.2018.462

\title{
Finite-sized rigid spheres in turbulent Taylor-Couette flow: effect on the overall drag
}

\author{
Dennis Bakhuis ${ }^{1}$, Ruben A. Verschoof ${ }^{1}$, Varghese Mathai ${ }^{1}$, \\ Sander G. Huisman ${ }^{1}$, Detlef Lohse ${ }^{1,2}$ and Chao Sun ${ }^{3,1,} \dagger$ \\ ${ }^{1}$ Physics of Fluids Group, Max Planck UT Center for Complex Fluid Dynamics, \\ MESA+ Institute and J.M. Burgers Centre for Fluid Dynamics, University of Twente, \\ P.O. Box 217, 7500 AE Enschede, The Netherlands \\ ${ }^{2}$ Max Planck Institute for Dynamics and Self-Organization, 37077 Göttingen, Germany \\ ${ }^{3}$ Center for Combustion Energy, Key Laboratory for Thermal Science and Power Engineering of Ministry \\ of Education, Department of Energy and Power Engineering, Tsinghua University, Beijing, China
}

(Received 7 December 2017; revised 17 April 2018; accepted 29 May 2018; first published online 4 July 2018)

We report on the modification of drag by neutrally buoyant spherical finite-sized particles in highly turbulent Taylor-Couette (TC) flow. These particles are used to disentangle the effects of size, deformability and volume fraction on the drag, and are contrasted to the drag in bubbly TC flow. From global torque measurements, we find that rigid spheres hardly decrease or increase the torque needed to drive the system. The size of the particles under investigation has a marginal effect on the drag, with smaller diameter particles showing only slightly lower drag. Increase of the particle volume fraction shows a net drag increase. However, this increase is much smaller than can be explained by the increase in apparent viscosity due to the particles. The increase in drag for increasing particle volume fraction is corroborated by performing laser Doppler anemometry, where we find that the turbulent velocity fluctuations also increase with increasing volume fraction. In contrast to rigid spheres, for bubbles, the effective drag reduction also increases with increasing Reynolds number. Bubbles are also much more effective in reducing the overall drag.

Key words: multiphase flow, drag reduction, shear layer turbulence

\section{Introduction}

Flows in nature and industry are generally turbulent, and often these flows carry bubbles, drops or particles of various shapes, sizes and densities. Examples include sediment-laden rivers, gas-liquid reactors, volcanic eruptions, plankton in the oceans, pollutants in the atmosphere and air bubbles in the ocean mixing layer (Toschi \& Bodenschatz 2009). Particle-laden flows may be characterized in terms of the particle density $\rho_{p}$, particle diameter $d_{p}$, volume fraction $\alpha$ and Reynolds number $R e$ of

$\dagger$ Email address for correspondence: chaosun@tsinghua.edu.cn 
the flow. When $d_{p}$ is small (compared with the dissipative length scale $\eta_{K}$ ) and $\alpha$ is low $\left(<10^{-3}\right)$, the system may be modelled using a point particle approximation with two-way coupling (Elghobashi 1994; Mazzitelli, Lohse \& Toschi 2003; Mathai et al. 2016). With recent advances in computing, fully resolved simulations of particle-laden flows have also become feasible. Uhlmann (2008) conducted one of the first numerical simulations of finite-sized rigid spheres in a vertical particle-laden channel flow. They observed a modification of the mean velocity profile and turbulence modulation due to the presence of particles. A number of studies followed, which employed immersed boundary (Peskin 2002; Cisse, Homann \& Bec 2013), Physalis (Naso \& Prosperetti 2010; Wang, Sierakowski \& Prosperetti 2017) and front-tracking methods (Unverdi \& Tryggvason 1992; Roghair et al. 2011; Tagawa et al. 2013) to treat rigid particles and deformable bubbles respectively in channel and pipe flow geometries (Pan \& Banerjee 1996; Lu, Fernández \& Tryggvason 2005; Uhlmann 2008; Dabiri, Lu \& Tryggvason 2013; Kidanemariam et al. 2013; Lashgari et al. 2014; Picano, Breugem \& Brandt 2015; Costa et al. 2016). Flows with dispersed particles, drops and bubbles can, under the right conditions, reduce the skin friction and result in significant energetic (and therefore financial) savings. In industrial settings, this is already achieved using polymeric additives which disrupt the self-sustaining cycle of wall turbulence and dampen the quasi-streamwise vortices (Procaccia, L'vov \& Benzi 2008; White \& Mungal 2008). Polymeric additives are impractical for maritime applications, and therefore gas bubbles are used, with varying success rates (Ceccio 2010; Murai 2014). Local measurements in bubbly flows are non-trivial, and the key parameters and their optimum values are still unknown. For example, it is impossible to fix the bubble size in experiments and therefore to isolate the effect of bubble size. Various studies have hinted that drag reduction can also be achieved using spherical particles (Zhao, Andersson \& Gillissen 2010) and also by using very large particles in a turbulent von Kármán flow (Cisse et al. 2015). In the latter study, a tremendous decrease in turbulent kinetic energy (TKE) was observed. A similar, but less intense, decrease in TKE was also seen by Bellani et al. (2012) using a very low particle volume fraction. By using solid particles, it is possible to isolate the size effect on drag reduction, and, even though rigid particles are fundamentally different from bubbles, this can give additional insight into the mechanism of bubbly drag reduction. Machicoane \& Volk (2016) have already shown that the particle dynamics is highly influenced by the diameter of the particle. This might or might not have a direct influence on the global drag of the system and has never been studied. Whether and when solid particles increase or decrease the drag in a flow is yet not fully understood, and two lines of thought exist. On one side, it is hypothesized that solid particles decrease the overall drag as they damp turbulent fluctuations (Poelma, Westerweel \& Ooms 2007; Zhao et al. 2010). On the other side, one could expect that solid particles increase the drag as they shed vortices, which must be dissipated. In addition, they also increase the apparent viscosity. A common way to quantify this is the so called 'Einstein relation' (Einstein 1906),

$$
v_{\alpha}=v\left(1+\frac{5}{2} \alpha\right)
$$

where $v$ is the viscosity of the continuous phase. This compensation is valid for the small $\alpha$ values used in this paper (Stickel \& Powell 2005). Direct measurements of drag in flows with solid particles are scarce, and the debate on under what conditions they either enhance or decrease the friction has not yet been settled. Particles and bubbles may show collective effects (clustering), and experiments have revealed that 
this has a significant influence on the flow properties (Liu \& Bankoff 1993; Kulick, Fessler \& Eaton 1994; Muste \& Patel 1997; So et al. 2002; Fujiwara, Minato \& Hishida 2004; van den Berg et al. 2005, 2007; Calzavarini et al. 2008; Shawkat, Ching \& Shoukri 2008; Colin, Fabre \& Kamp 2012; van Gils et al. 2013; Maryami et al. 2014; Mathai et al. 2015; Alméras et al. 2017; Mathai et al. 2018). In general, the Stokes number is used to predict this clustering behaviour, but for neutrally buoyant particles, this is found to be insufficient (Fiabane et al. 2012; Bragg, Ireland $\&$ Collins 2015). In addition, the position of the particles (or the particle clusters) is likely to have a large influence on the skin friction. In direct numerical simulation at low Reynolds numbers, Kazerooni et al. (2017) found that the particle distribution is mainly governed by the bulk Reynolds number.

In order to study the effects of particles on turbulence it is convenient to use a closed set-up where one can relate global and local quantities directly through rigorous mathematical relations. In this paper, the Taylor-Couette (TC) geometry (Grossmann, Lohse \& Sun 2016) - the flow between two concentric rotating cylinders - is employed, as this is a closed set-up with global balances. The driving of the TC geometry can be described using the Reynolds number based on the inner cylinder (IC), $R e_{i}=u_{i} d / v$, where $u_{i}=\omega_{i} r_{i}$ is the azimuthal velocity at the surface of the IC, $\omega_{i}$ is the angular velocity of the IC, $d=r_{o}-r_{i}$ is the gap between the cylinders, $v$ is the kinematic viscosity and $r_{i}\left(r_{o}\right)$ is the radius of the inner (outer) cylinder. The geometry of TC flow is characterized by two parameters: the radius ratio $\eta=r_{i} / r_{o}$ and the aspect ratio $\Gamma=L / d$, where $L$ is the height of the cylinders. The response parameter of the system is the torque, $\tau$, required to maintain constant rotation speed of the inner cylinder. It has been mathematically shown that in TC flow, the angular velocity flux, defined as $J^{\omega}=r^{3}\left(\left\langle u_{r} \omega\right\rangle_{A, t}-v(\partial / \partial r)\langle\omega\rangle_{A, t}\right)$, where the subscript $A, t$ denotes averaging over a cylindrical surface and time, is a radially conserved quantity (Eckhardt, Grossmann \& Lohse 2007). One can, in analogy to Rayleigh-Bénard convection, normalize this flux and define a Nusselt number based on the flux of the angular velocity,

$$
N u_{\omega}=\frac{J^{\omega}}{J_{\text {lam }}^{\omega}}=\frac{\tau}{2 \pi L \rho J_{\text {lam }}^{\omega}},
$$

where $J_{\text {lam }}^{\omega}=2 v r_{i}^{2} r_{o}^{2}\left(\omega_{i}-\omega_{o}\right) /\left(r_{o}^{2}-r_{i}^{2}\right)$ is the angular velocity flux for laminar purely azimuthal flow and $\omega_{o}$ is the angular velocity of the outer cylinder. In this spirit, the driving is expressed in terms of the Taylor number,

$$
T a=\frac{1}{4} \sigma d^{2}\left(r_{i}+r_{o}\right)^{2}\left(\omega_{i}-\omega_{o}\right)^{2} v^{-2} .
$$

Here, $\sigma=((1+\eta) /(2 \sqrt{\eta}))^{4} \approx 1.057$ is a geometric parameter ('geometric Prandtl number'), in analogy to the Prandtl number in Rayleigh-Bénard convection. In the presented work, where only the inner cylinder is rotated and the outer cylinder is kept stationary, we can relate $T a$ to the Reynolds number of the inner cylinder by

$$
R e_{i}=\frac{r_{i} \omega_{i} d}{v}=\frac{8 \eta^{2}}{(1+\eta)^{3}} \sqrt{T a} .
$$

The scaling of the dimensionless angular velocity flux (torque) with the Taylor (Reynolds) number has been analysed extensively, see, e.g., Lathrop, Fineberg \& Swinney (1992), Lewis \& Swinney (1999), van Gils et al. (2011), Paoletti \& Lathrop 


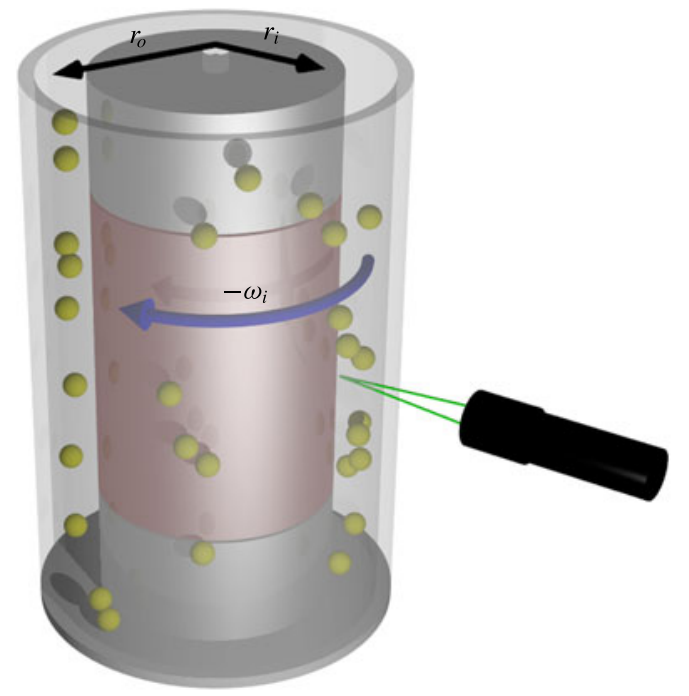

FIGURE 1. (Colour online) Schematic of the TC set-up: two concentric cylinders of radii $r_{i, o}$ with a working fluid in between. Particles are not to scale. The inner cylinder rotates with angular velocity $\omega_{i}$, while the outer cylinder is kept at rest. We measure the torque on the middle section (highlighted). The laser Doppler anemometry (LDA) probe is positioned at midheight to measure the azimuthal velocity at midgap.

(2011), Ostilla-Mónico et al. (2013) and the review articles by Fardin, Perge \& Taberlet (2014) and Grossmann et al. (2016), and the different regimes are well understood. In the current Taylor number regime, it is known that $N u_{\omega} \propto T a^{0.4}$. Because this response is well known, it can be exploited to study the influence of immersed bubbles and particles (van den Berg et al. 2005, 2007; van Gils et al. 2013; Maryami et al. 2014; Verschoof et al. 2016) on the drag needed to sustain constant rotational velocity of the inner cylinder.

In this paper, we will use the TC geometry to study the effect of neutrally buoyant rigid spherical particles on the drag. We study the effects of varying the particle size $d_{p}$, the volume fraction $\alpha$, the density ratio $\phi$ and the flow Reynolds number $R e$ on the global torque (drag) of the TC flow. The drag reduction is expressed as DR = $\left(1-N u_{\omega}(\alpha) / N u_{\omega}(\alpha=0)\right)$ and, as we are interested in the net drag reduction, it is not compensated for increased viscosity effects using correction models, such as the Einstein relation.

The paper is organized as follows. Section 2 presents the experimental set-up. In $\S 3$, we discuss the results. The findings are summarized and an outlook for future work is given in the last section.

\section{Experimental set-up}

The experiments were conducted in the Twente Turbulent Taylor-Couette $\left(\mathrm{T}^{3} \mathrm{C}\right)$ facility (van Gils et al. 2011). A schematic of the set-up is shown in figure 1. In this set-up, the flow is confined between two concentric cylinders, which rotate independently. The top and bottom plates are attached to the outer cylinder. The radius of the inner cylinder (IC) is $r_{i}=0.200 \mathrm{~m}$ and the radius of the outer cylinder (OC) is $r_{o}=0.2794 \mathrm{~m}$, resulting in a gap width of $d=r_{o}-r_{i}=0.0794 \mathrm{~m}$ and a 
radius ratio of $\eta=r_{i} / r_{o}=0.716$. The IC has a total height of $L=0.927 \mathrm{~m}$, resulting in an aspect ratio of $L / d=11.7$. The IC is segmented axially into three parts. To minimize the effect of the stationary end plates, the torque is measured only over the middle section of the IC with height $L_{\text {mid }} / L=0.58$, away from the end plates. A hollow reaction torque sensor made by Honeywell is used to measure the torque, which has an error of roughly $1 \%$ for the largest torques we measured. Between the middle section and the top and bottom sections of the inner cylinder is a gap of $2 \mathrm{~mm}$.

The IC can be rotated up to $f_{i}=\omega_{i} /(2 \pi)=20 \mathrm{~Hz}$. In these experiments, only the IC is rotated and the OC is kept at rest. The system holds a volume of $V=111 \mathrm{l}$ of working fluid, which is a solution of glycerol $\left(\rho=1260 \mathrm{~kg} \mathrm{~m}^{-3}\right)$ and water. To tune the density of the working fluid, the amount of glycerol is varied between $0 \%$ and $40 \%$, resulting in particles being marginally heavy, neutrally buoyant or marginally light. The system is thermally controlled by cooling the top and bottom plates of the set-up. The temperature is kept at $T=(20 \pm 1)^{\circ} \mathrm{C}$ for all the experiments, with a maximum spatial temperature difference of $0.2 \mathrm{~K}$ within the set-up, and we account for the density and viscosity changes of water and glycerol (Glycerine Producers' Association 1963).

Rigid polystyrene spherical particles (RGPballs S.r.l.) are used in the experiments; these particles have a density close to that of water $\left(940-1040 \mathrm{~kg} \mathrm{~m}^{-3}\right)$. We chose particles with diameters $d_{p}=1.5,4.0$ and $8.0 \mathrm{~mm}$. At our disposal are 2.221 of $1.5 \mathrm{~mm}$ diameter particles, 2.221 of $4 \mathrm{~mm}$ diameter particles and 6.661 of $8 \mathrm{~mm}$ diameter particles, resulting in maximum volume fractions of $2 \%, 2 \%$ and $6 \%$ respectively. The particles are found to be nearly mono-disperse $(99.9 \%$ of the particles are within $\pm 0.1 \mathrm{~mm}$ of their target diameter). Due to the fabrication process, small air bubbles are sometimes entrapped within the particles. This results in a slightly heterogeneous density distribution of the particles. After measuring the density distribution for each diameter, we calculated the average for all batches, which was $\rho_{p}=1036 \pm 5 \mathrm{~kg} \mathrm{~m}^{-3}$. By adding glycerol to water, we match this value in order to have neutrally buoyant particles.

Using a laser Doppler anemometry (LDA) system (BSA F80, Dantec Dynamics) we capture the azimuthal velocity at midheight and midgap of the system (see figure 1) and we perform a radial scan at midheight. The flow is seeded with $5 \mu \mathrm{m}$ diameter polyamide particles (PSP-5, Dantec Dynamics). Because of the curved surface of the outer cylinder (OC), the beams of the LDA get refracted in a non-trivial manner, which is corrected for using a ray-tracing technique described by Huisman, van Gils \& Sun (2012).

Obviously, LDA measurements in a multiphase flow are more difficult to set up than for single-phase flows, as the method relies on the reflection of light from tiny tracer particles passing through a measurement volume $(0.07 \mathrm{~mm} \times 0.07 \mathrm{~mm} \times 0.3 \mathrm{~mm})$. Once we add a second type of relatively large particles to the flow, this will affect the LDA measurements, mostly by blocking the optical path, resulting in lower acquisition rates. These large particles will also move through the measurement volume, but as these particles are at least 300 times larger than the tracers and thus much larger than the fringe pattern (fringe spacing $d_{f}=3.4 \mu \mathrm{m}$ ), the reflected light is substantially different from a regular Doppler burst and does not result in a measured value. The minimal signal-to-noise ratio for accepting a Doppler burst is set to 4 . As a postprocessing step, the velocities are corrected for the velocity bias by using the transit time of the tracer particle. 
(a)

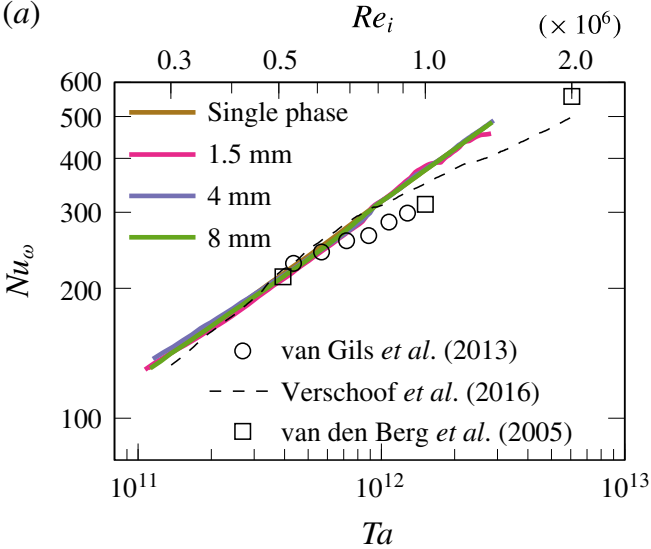

(b)
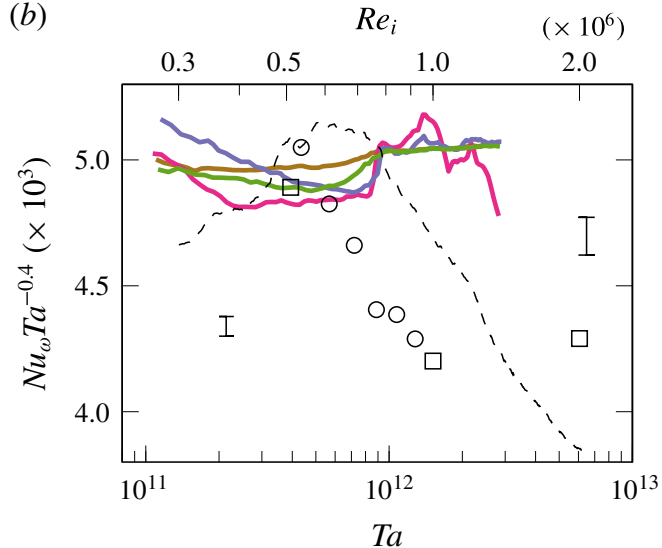

FIgURE 2. (Colour online) (a) A plot of $N u_{\omega}(T a)$ for $2 \%$ particle volume fraction with particle diameters of $1.5 \mathrm{~mm}, 4.0 \mathrm{~mm}$ and $8.0 \mathrm{~mm}$, and for comparison the single-phase case. Data from comparable bubbly drag reduction studies are plotted using black markers. (b) The same data, but now as a compensated plot $N u_{\omega} / T a^{0.40}$ as a function of $T a$. The error bar indicates the maximum deviation for repeated measurements from all measurements combined (coloured curves), which is less than $1 \%$. At $T a \geqslant 2 \times 10^{12}$, the $1.5 \mathrm{~mm}$ particles show an increased uncertainty of $1.7 \%$, which is indicated by the right error bar.

\section{Results}

\subsection{Effect of particle size}

First, we study the effect of changing the particle diameter on the torque of the system. In these experiments, we kept the particle volume fraction fixed at $2 \%$ and the density of the working fluid, $\rho_{f}$, at $1036 \mathrm{~kg} \mathrm{~m}^{-3}$, for which the particles are neutrally buoyant. The results of these measurements are presented as $N u_{\omega}(T a)$ in figure 2(a). Our curves are practically overlapping, suggesting that the difference in drag between the different particle sizes is only marginal. We compare these with the bubbly drag reduction data at similar conditions (hollow symbols) from van den Berg et al. (2005), van Gils et al. (2013) and Verschoof et al. (2016). At low Ta, the symbols overlap with our data. However, at larger $T a$, the bubbly flow data show much lower torque (drag) than the particle-laden cases. As we are in the ultimate regime of turbulence where $N u_{\omega}$ effectively scales as $N u_{\omega} \propto T a^{0.4}$ (Huisman et al. 2012; Ostilla-Mónico et al. 2013), we compensate the data with $T a^{0.40}$ in figure 2(b) to emphasize the differences between the datasets. For the single-phase case, this yields a clear plateau. For the particle-laden cases, the lowest drag corresponds to the smallest particle size. The reduction is, however, quite small $(<3 \%)$. The compensated plots also reveal a sudden increase in drag at a critical Taylor number of $\mathrm{Ta}^{*}=0.8 \times 10^{12}$. The jump is more distinct for the smaller particles, and might suggest a reorganization of the flow (Huisman et al. 2014). Beyond $T a^{*}$, the drag reduction is negligible for the larger particles (4 $\mathrm{mm}$ and $8 \mathrm{~mm}$ spheres). However, for the $1.5 \mathrm{~mm}$ particles, the drag reduction seems to increase, and was found to be very repeatable in experiments. Interestingly, the size of these particles is comparable to that of the air bubbles in van Gils et al. (2013). This might suggest that for smaller size particles at larger $T a$, one could expect drag reduction. At the increased viscosity of the suspension, a maximum $T a \approx 3 \times 10^{12}$ could be reached in our experiments. 


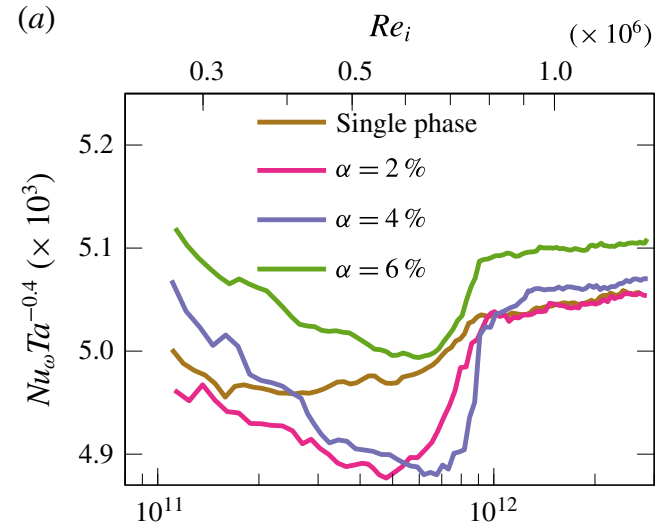

$\mathrm{Ta}$ (b)

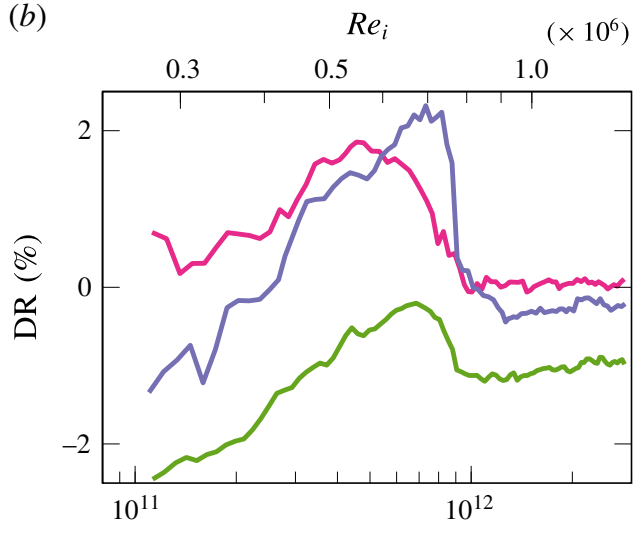

$\mathrm{Ta}$

Figure 3. (Colour online) (a) A plot of $N u_{\omega}(T a)$, compensated by $T a^{0.4}$, for $8 \mathrm{~mm}$ particles with various particle volume fractions, and for comparison the single-phase case. (b) The drag reduction, defined as $\mathrm{DR}=\left(1-N u_{\omega}(\alpha) / N u_{\omega}(\alpha=0)\right)$, plotted against $T a$.

We have performed an uncertainty analysis by repeating the measurements for the single phase and for the cases with $8 \mathrm{~mm}$ and $1.5 \mathrm{~mm}$ particles multiple times and calculating the maximum deviation from the ensemble average. The left error bar indicates the maximum deviation for all measurements combined and is $\approx 1 \%$. For $T a \geqslant 2 \times 10^{12}$, we see an increase in uncertainty of $1.7 \%$ (shown by the right error bar in figure $2 b$ ), which is only caused by the $1.5 \mathrm{~mm}$ particles. These tiny particles can accumulate in the $2 \mathrm{~mm}$ gap between the cylinder segments and thereby increase the uncertainty. Above $T a \geqslant 2 \times 10^{12}$, both the $8 \mathrm{~mm}$ and $4 \mathrm{~mm}$ particles show a maximum deviation below $0.25 \%$.

Below $\mathrm{Ta}^{*}$, the drag reduction due to spherical particles appears to be similar to bubbly drag reduction (van Gils et al. 2013). However, in the lower- $T a$ regime, the bubble distribution is highly non-uniform due to the buoyancy of the bubbles (van den Berg et al. 2005; van Gils et al. 2013; Verschoof et al. 2016). Therefore, the volume fractions reported are only the global values, and the torque measurements are for the midsections of their set-ups. What is evident from the above comparisons is that in the high- $\mathrm{Ta}$ regime, air bubbles drastically reduce the drag, reaching far beyond the drag modification by rigid spheres.

\subsection{Effect of particle volume fraction}

The next step is to investigate the effect of the particle volume fraction on the torque. For the $8 \mathrm{~mm}$ particles, we have the ability to increase the particle volume fraction up to $6 \%$. This is done in steps of $2 \%$, and the results are plotted in compensated form in figure $3(a)$. The normalized torque increases with the volume fraction of particles. The $6 \%$ case shows the largest drag. Figure 3(b) shows the same data in terms of drag reduction as function of $\mathrm{Ta}$. A $2 \%$ volume fraction of particles gives the highest drag reduction. With increasing $\alpha$, the drag reduction decreases. These measurements are in contrast to the findings for bubbly drag reduction (van Gils et al. 2013), for which the net drag decrease with increasing gas volume fraction of drag in a particle-laden flow is the larger apparent viscosity. If we were to calculate the drag modification using an apparent viscosity (e.g. the Einstein relation (1.1)) for the case using $\alpha=6 \%$ 

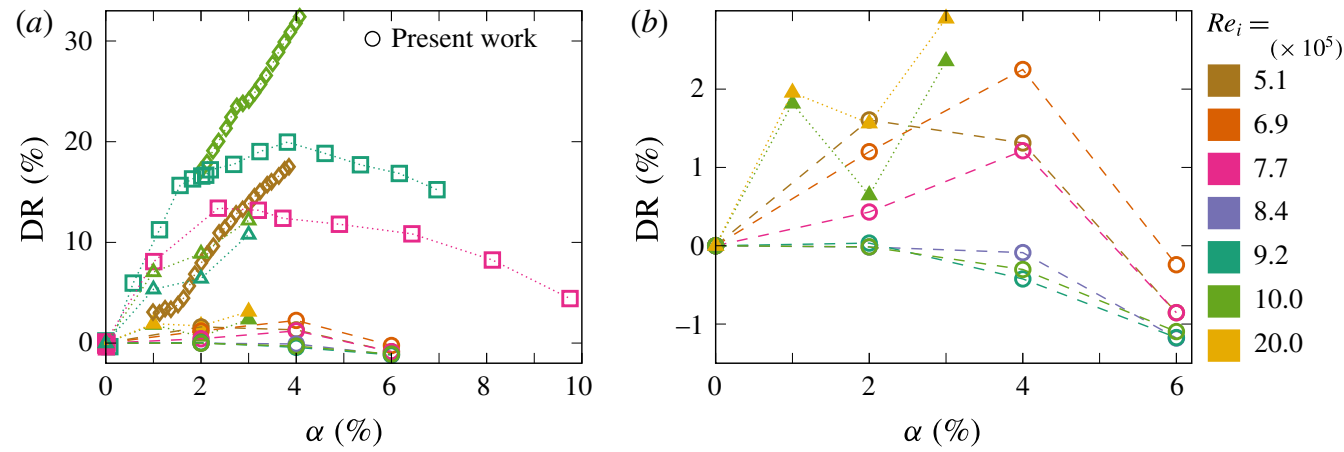

FIgURE 4. (Colour online) (a) Drag reduction as function of particle volume fraction from $\bigcirc d_{p}=8 \mathrm{~mm}$ particles from the present work compared with similar gas volume fractions from $\square$ van den Berg et al. (2005), $\diamond$ van Gils et al. (2013) and $\Delta$ Verschoof et al. (2016). Symbols indicate the different studies while colours differentiate between the Reynolds numbers. The current work has DR defined as $\left(1-N u_{\omega}(\alpha) / N u_{\omega}(\alpha=0)\right)$; the other studies use dimensionless torque $G$ (van Gils et al. 2013), friction coefficient $c_{f}$ (van den Berg et al. 2005) or plain torque $\tau$ (Verschoof et al. 2016) to define DR. (b) Zoom of the bottom part of $(a)$ where the data from the present work are compared with bubbly drag reduction data using 6 ppm of surfactant from Verschoof et al. (2016).

of particles, the measured drag would be $15 \%$ larger compared to the pure working fluid case. Inclusion of this effect in our drag reduction calculation would result in reductions of the same order. However, when comparing the drag with or without particles, the net drag reduction is practically zero. This result is different from the work of Picano et al. (2015) in turbulent channel flow, where they found that the drag increased more than the increase of the viscosity.

For a better comparison with bubbly drag reduction, we plot the drag reduction as a function of (gas or particle) volume fraction $\alpha$; see figure 4(a). Different studies are shown using different symbols, and $R e$ is indicated by colours. None of the datasets were compensated for the changes in effective viscosity. The DR is defined in a slightly different way in each study: van den Berg et al. (2005) makes use of the friction coefficient $\left(1-c_{f}(\alpha) / c_{f}(0)\right)$; van Gils et al. (2013) uses the dimensionless torque $G=\tau /\left(2 \pi L_{\text {mid }} \rho v^{2}\right),(1-G(\alpha) / G(0))$; Verschoof et al. (2016) uses the plain torque value $(1-\tau(\alpha) / \tau(0))$. While the rigid particles only show marginal drag reduction, some studies using bubbles achieve dramatic reduction of up to $30 \%$ and beyond. Figure $4(b)$ shows a zoomed in view of the bottom part of the plot with the rigid-sphere data. The triangles denote the data from Verschoof et al. (2016), corresponding to small bubbles in the TC system. The rigid particles and the small bubbles show a similar drag response. What is remarkable is that this occurs despite the huge difference in size. The estimated diameter of the bubbles in Verschoof et al. (2016) is $0.1 \mathrm{~mm}$, while the rigid spheres are approximately two orders in magnitude larger. This provides key evidence that the particle size alone is not enough to cause drag reduction, the density ratio of the particles and the carrier fluid is also of importance.

\subsection{Effect of marginal changes in particle density ratio}

With the effects of particle size and volume fraction revealed, we next address the sensitivity of the drag to marginal variations in particle density. A change in the 
(a)

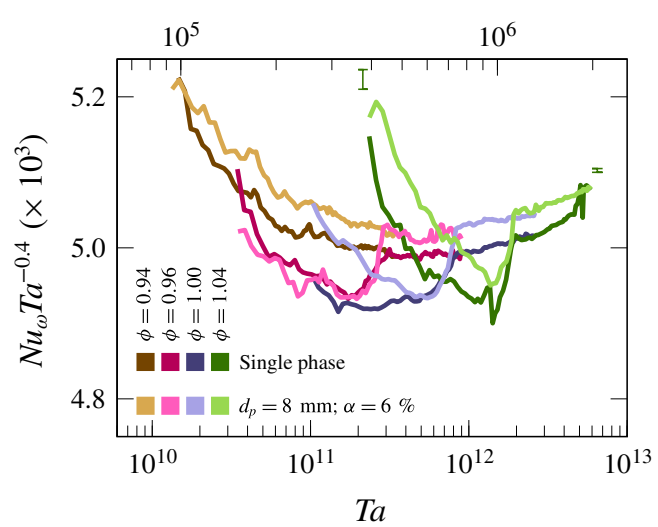

(b)

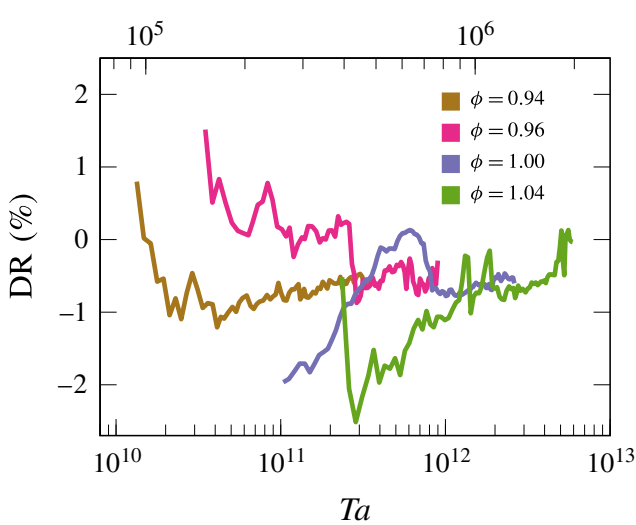

FIgure 5. (Colour online) (a) A plot of $T a$ as a function of $N u_{\omega}$ compensated by $T a^{0.4}$ for various density ratios $\phi=\rho_{p} / \rho_{f}$ indicated by the corresponding colour. The darker shades indicate the single-phase cases while the lighter shades show the cases using $6 \%$ particle volume fraction of $8 \mathrm{~mm}$ diameter particles. Due to the increase in viscosity, the maximum attainable $T a$ is lower for larger density ratios. The uncertainty is again estimated using the maximum deviation from the average for multiple runs and here is only shown for the green curves. This value is slightly below $1 \%$ at lower $T a$ and decreases with increasing $T a$ to values below $0.25 \%$. This trend is seen for all values of $\phi$. (b) The drag reduction, calculated from the data of $(a)$, plotted against Ta. The drag reduction is defined as $\mathrm{DR}=\left(1-N u_{\omega}(\alpha=6 \%) / N u_{\omega}(\alpha=0)\right)$.

particle density ratio brings about a change in the buoyancy and centrifugal forces on the particle, both of which can affect the particle distribution within the flow. We tune the particle to fluid density ratio $\phi \equiv \rho_{p} / \rho_{f}$ by changing the volume fraction of glycerol in the fluid, such that the particles are marginally buoyant $(\phi=0.94,0.97)$, neutrally buoyant $(\phi=1.00)$ and marginally heavy $(\phi=1.04)$ particles. In figure 5(a), we show the compensated $N u_{\omega}$ as a function of $T a$ for various values of $\phi$. Here, $\alpha$ is fixed to $6 \%$ and only $8 \mathrm{~mm}$ particles are used. The darker shades of colour correspond to the single-phase cases, while lighter shades correspond to particle-laden cases. In general, the single-phase drag is larger as compared with the particle-laden cases. However, there is no striking difference between the different values of $\phi$. In figure $5(b)$, we present the drag reduction for particle-laden cases at different density ratios. On average, we see for all cases drag modification of approximately $\pm 2 \%$. We can also identify a small trend in the lower- $T a$ region: the two larger $\phi$ cases (heavy and neutrally buoyant particles) tend to have a drag increase, while the smaller $\phi$ cases (both light particles) have a tendency for drag reduction. Nevertheless, the absolute difference in DR between the cases is within $4 \%$. The above results provide clear evidence that minor density mismatches do not have a serious influence on the global drag of the system. To investigate for strong buoyancy effects, additional measurements were made using $2 \mathrm{~mm}$ expanded polystyrene particles $(\phi=0.02)$. However, due to the particles accumulating between the inner cylinder segments leading to additional mechanical friction, these measurements were inconclusive.

\subsection{Flow statistics using particles}

In the above sections, we presented the effects of changing particle size, volume fraction and density on the global drag of the system. Next, we look into local 

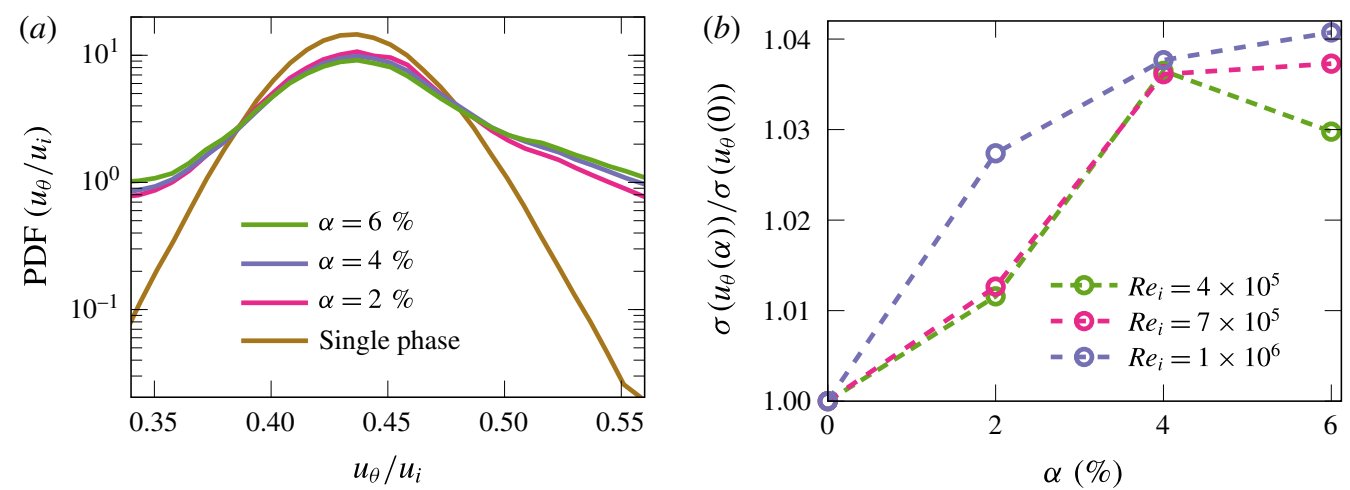

FIGURE 6. (Colour online) (a) Probability density functions of $u_{\theta} / u_{i}$ for various values of $\alpha$ and the single-phase case. The particle size is fixed to $8 \mathrm{~mm}$ and $R e_{i}=1 \times 10^{6}$ for all cases. (b) Standard deviation of the azimuthal velocity normalized by the standard deviation of the single-phase case for three different values of $R e$ for a fixed particle size of $8 \mathrm{~mm}$.

flow properties using LDA while the particles are present. First, we collect a total of $1 \times 10^{6}$ data points of azimuthal velocity at midheight and midgap. These are captured over a period of approximately $3 \times 10^{4}$ cylinder rotations. From these data, we calculate the probability density function (PDF) of $u_{\theta}$ normalized by $u_{i}$ for various values of $\alpha$, shown in figure 6(a). The particle size is fixed to $8 \mathrm{~mm}$ and the Reynolds number is set to $1 \times 10^{6}$. From this figure, we see a large increase in turbulent fluctuations, resulting in very wide tails. While the difference between $2 \%$, $4 \%$ and $6 \%$ is not large, we can identify an increase in fluctuations with increasing $\alpha$. These increased fluctuations can be explained by the additional wakes produced by the particles (Poelma et al. 2007; Alméras et al. 2017). The increase in fluctuations can also be visualized using the standard deviation of $\sigma\left(u_{\theta}\right)=\left\langle u_{\theta}^{\prime 2}\right\rangle^{1 / 2}$ normalized by the standard deviation of the single-phase case - see figure $6(b)$. In this figure, $\sigma\left(u_{\theta}\right)$ is shown for three different values of $R e$, again for $8 \mathrm{~mm}$ particles. In general, we see a monotonically increasing trend with $\alpha$, and it seems to approach an asymptotic value. One can speculate that there has to be an upper limit for fluctuations that originate from wakes of the particles. For large $\alpha$, the wakes from particles will interact with one another and with the carried flow.

Measurements using $4 \mathrm{~mm}$ particles yielded qualitatively similar results. It is known that in particle-laden gaseous pipe flows, large particles can increase the turbulent fluctuations, while small particles result in turbulence attenuation (Tsuji, Morikawa \& Shiomi 1984; Gore \& Crowe 1989; Vreman 2015). The LDA measurements were not possible with the smallest particles $(1.5 \mathrm{~mm})$, as the large amount of particles in the flow blocked the optical paths of the laser beams.

We are confident that for these bidisperse particle-laden LDA measurements, the large particles do not have an influence on the measurements as these millimetric-sized particles are much larger than the fringe spacing $\left(d_{f}=3.4 \mu \mathrm{m}\right)$ and do not show a Doppler burst. However, during the measurements, the particles get damaged and small bits of material are fragmented off the particles. We estimate the size of these particles to be slightly larger than the tracer particles, and these can have an influence on the LDA measurements as they do not act as tracers. 

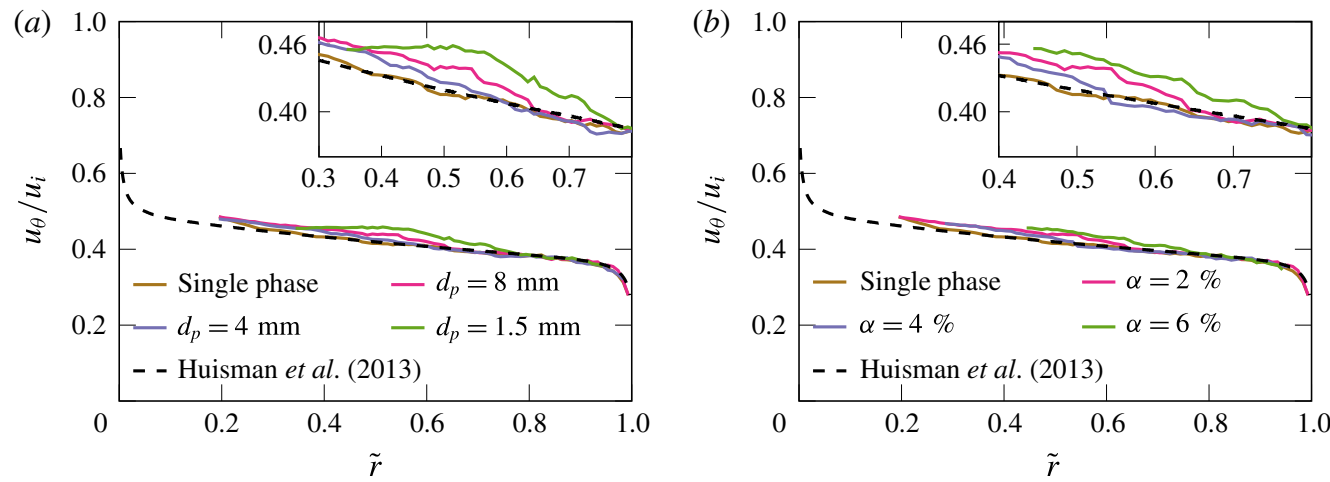

FIgURE 7. (Colour online) A plot of $u_{\theta}$ normalized by the velocity of the inner cylinder wall $u_{i}$ as a function of the normalized radius for various values $d_{p}$ while $\alpha=2 \%$ (a) and various values of $\alpha$ while $d_{p}=8 \mathrm{~mm}(b)$. In all cases, $R e_{i}$ is fixed to $1 \times 10^{6}$. For comparison, the single-phase case using water at $R e_{i}=1 \times 10^{6}$ from Huisman et al. (2013) is also plotted in dashed black in both plots. Both plots have an inset showing an enlargement of the centre area from the same plot.

The way in which the average azimuthal velocity changes with particle radius is shown in figure 7 . We measured a total of $3 \times 10^{4}$ data points during approximately 900 cylinder rotations. Again, the data are corrected for velocity bias by using the transit time as a weighing factor. Figure $7(a)$ shows the effect of particle size for $\alpha=2 \%$ and figure 7(b) shows the effect of particle volume fraction for $8 \mathrm{~mm}$ particles. Both figures additionally show the high-precision single-phase data from Huisman et al. (2013), for which our single-phase measurements are practically overlapping. Since LDA measurements close to the inner cylinder are difficult, due to the reflecting inner cylinder surface, we limited our radial extent to $\tilde{r}=\left(r-r_{i}\right) /\left(r_{o}-r_{i}\right)=[0.2,1]$. We found that the penetration depth of our LDA measurements is the smallest for experiments with the smallest particles and the largest $\alpha$. All differences from the single-phase case are only marginal and we can conclude that the average mean velocity is not much affected by the particles in the flow, at least for $\tilde{r} \geqslant 0.2$.

To get an idea of the fluctuations we can use the previous data to construct twodimensional PDFs of the azimuthal velocity as a function of radius. These are shown for $R e=1 \times 10^{6}$ using $8 \mathrm{~mm}$ particles at various values of $\alpha$ and the single-phase case in figure 8 . The first thing to notice is again that the penetration depth decreases with increasing $\alpha$. The single-phase case shows a narrow-banded PDF. When $\alpha$ is increased, for the lower values of $\tilde{r}$, the PDF is much wider. While it makes sense that an increase in $\alpha$ increases the fluctuations due to the increased number of wakes of particles, this is expected everywhere in the flow, not only closer to the inner cylinder. It is possible that the particles have a preferred concentration closer to the inner cylinder. We tried to measure the local concentration of particles as a function of radius but failed due to limited optical accessibility. Therefore, we can only speculate under what circumstances there would be an inhomogeneous particle distribution that would lead to the visible increase in fluctuations. The first possibility is a mismatch in density between the particles and the fluid, which would result in light particles $(\phi<1)$ accumulating closer to the inner cylinder. Another possibility is that due to the rotation of the particles, an effective lift force arises, leading to a different particle 

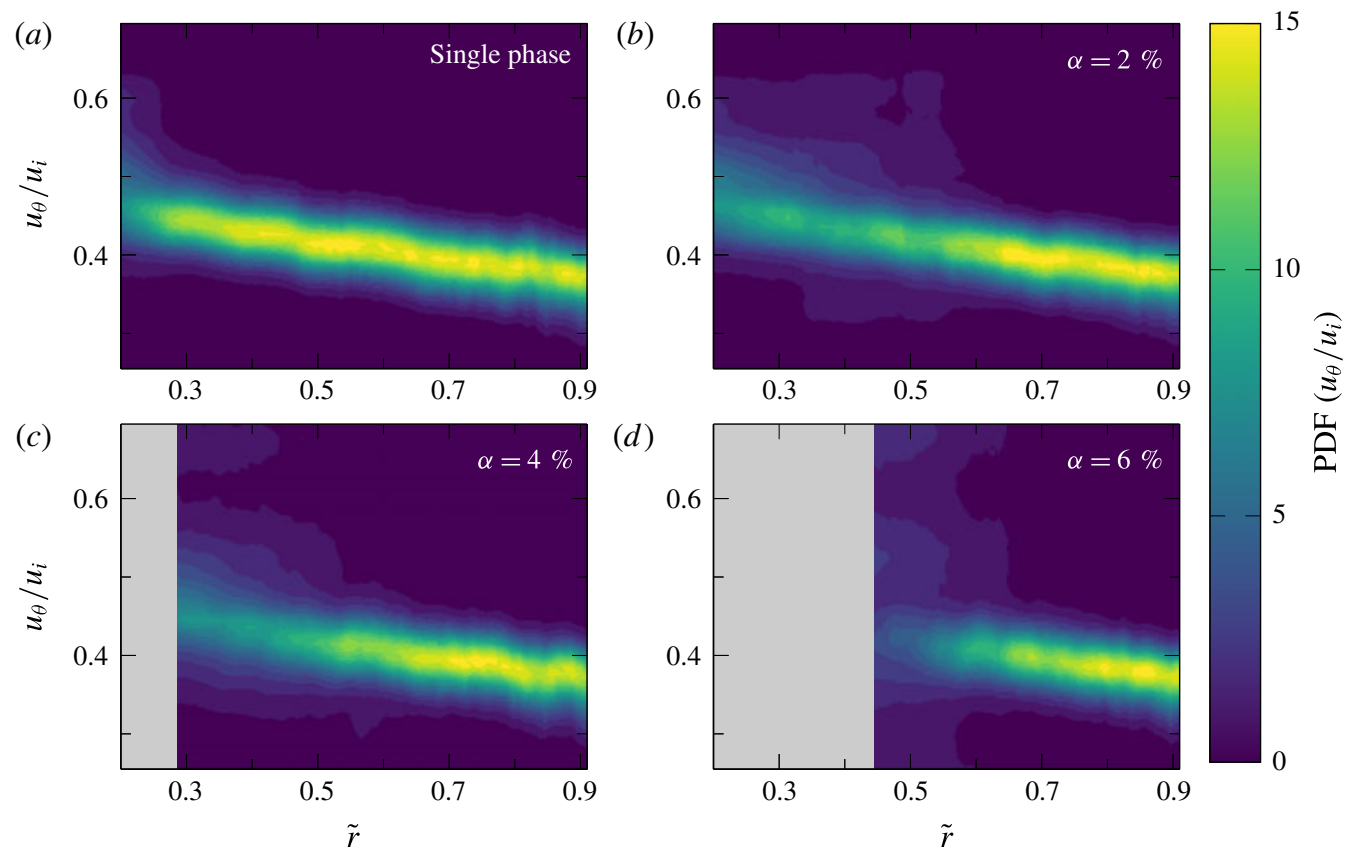

FIGURE 8. (Colour online) Probability density functions of the normalized azimuthal velocity as a function of the normalized radial position for various values of $\alpha$ for the case of $8 \mathrm{~mm}$ particles and the single-phase case while keeping $R e$ at $1 \times 10^{6}$. With increasing $\alpha$, the maximum penetration depth decreases. The grey areas indicate radial positions for which no data are available.

distribution in the flow. While this is quite plausible, this is difficult to validate as we would need to capture the rotation. The fragments of plastic that are sheared off the particles can also give a bias to the LDA measurement. While we estimate them to be larger than the tracers, they might still be small enough to produce a signal, and they might not follow the flow faithfully.

\section{Conclusions and outlook}

We have conducted an experimental study on the drag response of a highly turbulent TC flow containing rigid neutrally buoyant spherical particles. We have found that, unlike the case of bubbles used in prior works (van Gils et al. 2013; Verschoof et al. 2016), rigid particles barely reduce (or increase) the drag on the system, even for cases where their size is comparable to that of the bubbles used in other studies. There is no significant size effect. Even for very large particles, which can attenuate turbulent fluctuations and generate wakes, there is no distinct difference from the single-phase flow. We also varied the volume fraction of the particles in the range $0 \%-6 \%$. The particle volume fraction has no greater effect on the system drag than what is expected due to changes in the apparent viscosity of the suspension. Further, we tested the sensitivity of our drag measurements to marginal variations in the particle to fluid density ratio $\phi$. A trend was noticeable, towards drag reduction when $\phi$ was reduced from 1.00 to 0.94 . This suggests that a low density of the particles could be a necessary ingredient for drag reduction. Finally, we have also 
probed the local flow at the midheight and midgap of the system using LDA. With the addition of particles, the liquid velocity fluctuations are enhanced, with wider tails of the distributions. A finite relative velocity between the particle and the flow around it can cause this increase in velocity fluctuations (Mathai et al. 2015), as seen for bubbly flows (pseudo-turbulence) and in situations of sedimenting particles in quiescent or turbulent environments (Gore \& Crowe 1989). In the present situation, the relative velocity between the particle and the flow is expected, due to the inertia of the finite-sized particles we used. There is only a marginal deviation from the single-phase case in the average azimuthal velocity over the radial positions measured using any size or concentration of particles measured. From the two-dimensional PDFs, we see that closer to the inner cylinder, using smaller $d_{f}$ or larger $\alpha$, the PDF gets wider. This could be due to a preferential concentration of the particles or a slight density mismatch.

Our study is a step towards a better understanding of the mechanisms of bubbly drag reduction. Bubbles are deformable, and they have a tendency to migrate towards the walls, either due to lift force (Dabiri et al. 2013) or due to centripetal effects (van Gils et al. 2013). When compared with the drag reducing bubbles in van Gils et al. (2013) and Verschoof et al. (2016), our particles do not deform, and they do not experience centripetal effects as they are density matched. At least one of these differences must therefore be crucial for the observed bubbly drag reduction in those experiments. In a future investigation, we will conduct more experiments using very light spherical particles that experience similar centripetal forces to the bubbles in van Gils et al. (2013), but are non-deformable. These particles need to be larger than the size of the gap between the inner cylinder segments, and very rigid, or the set-up needs to be modified to close the gap between the IC segments. Such experiments can then disentangle the role of particle density on drag reduction from that of the particle shape.

\section{Acknowledgements}

We would like to thank E. Guazzelli, B. Vreman, R. Ezeta, P. Bullee and A. te Nijenhuis for various stimulating discussions. Moreover, we would like to thank G.-W. Bruggert and M. Bos for technical support. This work was funded by the Natural Science Foundation of China under grant no. 11672156, VIDI grant no. 13477, STW, FOM and MCEC, which are part of the Netherlands Organisation for Scientific Research (NWO).

\section{REFERENCES}

Alméras, E., Mathai, V., Lohse, D. \& Sun, C. 2017 Experimental investigation of the turbulence induced by a bubble swarm rising within incident turbulence. J. Fluid Mech. 825, 1091-1112.

Bellani, G., Byron, M. L., Collignon, A. G., Meyer, C. R. \& Variano, E. A. 2012 Shape effects on turbulent modulation by large nearly neutrally buoyant particles. J. Fluid Mech. 712, 41-60.

van den Berg, T. H., van Gils, D. P. M., Lathrop, D. P. \& Lohse, D. 2007 Bubbly turbulent drag reduction is a boundary layer effect. Phys. Rev. Lett. 98, 084501.

van Den Berg, T. H., Luther, S., Lathrop, D. P. \& Lohse, D. 2005 Drag reduction in bubbly Taylor-Couette turbulence. Phys. Rev. Lett. 94, 044501.

BragG, A. D., Ireland, P. J. \& Collins, L. R. 2015 Mechanisms for the clustering of inertial particles in the inertial range of isotropic turbulence. Phys. Rev. E 92 (2), 023029. 
Calzavarini, E., Cencini, M., Lohse, D. \& Toschi, F. 2008 Quantifying turbulence-induced segregation of inertial particles. Phys. Rev. Lett. 101 (8), 084504.

Ceccio, S. L. 2010 Friction drag reduction of external flows with bubble and gas injection. Annu. Rev. Fluid Mech. 42, 183-203.

Cisse, M., Homann, H. \& Bec, J. 2013 Slipping motion of large neutrally buoyant particles in turbulence. J. Fluid Mech. 735, R1.

Cisse, M., Saw, E.-W., Gibert, M., Bodenschatz, E. \& Bec, J. 2015 Turbulence attenuation by large neutrally buoyant particles. Phys. Fluids 27, 061702.

Colin, C., FABRE, J. \& KAMP, A. 2012 Turbulent bubbly flow in pipe under gravity and microgravity conditions. J. Fluid Mech. 711, 469-515.

Costa, P., Picano, F., Brandt, L. \& Breugem, W.-P. 2016 Universal scaling laws for dense particle suspensions in turbulent wall-bounded flows. Phys. Rev. Lett. 117, 134501.

Dabiri, S., Lu, J. \& Tryggvason, G. 2013 Transition between regimes of a vertical channel bubbly upflow due to bubble deformability. Phys. Fluids 25, 102110.

Eckhardt, B., Grossmann, S. \& Lohse, D. 2007 Torque scaling in turbulent Taylor-Couette flow between independently rotating cylinders. J. Fluid Mech. 581, 221-250.

Einstein, A. 1906 Eine neue Bestimmung der Moleküldimensionen. Ann. Phys. 324 (2), 289-306.

Elghobashi, S. 1994 On predicting particle-laden turbulent flows. Appl. Sci. Res. 52 (4), 309-329.

Fardin, M. A., Perge, C. \& Taberlet, N. 2014 The hydrogen atom of fluid dynamics introduction to the Taylor-Couette flow for soft matter scientists. Soft Matt. 10, 3523-3535.

Fiabane, L., Zimmermann, R., Volk, R., Pinton, J.-F. \& Bourgoin, M. 2012 Clustering of finite-size particles in turbulence. Phys. Rev. E 86 (3), 035301.

Fujiwara, A., Minato, D. \& Hishida, K. 2004 Effect of bubble diameter on modification of turbulence in an upward pipe flow. Intl J. Heat Fluid Flow 25 (3), 481-488.

van Gils, D. P., Bruggert, G.-W., Lathrop, D. P., Sun, C. \& Lohse, D. 2011 The Twente Turbulent Taylor-Couette $\left(\mathrm{T}^{3} \mathrm{C}\right)$ facility: strongly turbulent (multi-phase) flow between independently rotating cylinders. Rev. Sci. Instrum. 82, 025105.

van Gils, D. P., Narezo Guzman, D., Sun, C. \& Lohse, D. 2013 The importance of bubble deformability for strong drag reduction in bubbly turbulent Taylor-Couette flow. J. Fluid Mech. 722, 317-347.

Glycerine Producers' Association 1963 Physical Properties of Glycerine and its Solutions. Glycerine Producers' Association.

Gore, R. A. \& Crowe, C. T. 1989 Effect of particle size on modulating turbulent intensity. Intl J. Multiphase Flow 15 (2), 279-285.

Grossmann, S., Lohse, D. \& Sun, C. 2016 High-Reynolds number Taylor-Couette turbulence. Annu. Rev. Fluid Mech. 48, 53-80.

Huisman, S. G., van Gils, D. P. \& Sun, C. 2012 Applying laser Doppler anemometry inside a Taylor-Couette geometry using a ray-tracer to correct for curvature effects. Eur. J. Mech. (B/Fluids) 36, 115-119.

Huisman, S. G., Scharnowski, S., Cierpka, C., Kähler, C. J., Lohse, D. \& Sun, C. 2013 Logarithmic boundary layers in strong Taylor-Couette turbulence. Phys. Rev. Lett. 110, 264501.

Huisman, S. G., van Der Veen, R. C., Sun, C. \& Lohse, D. 2014 Multiple states in highly turbulent Taylor-Couette flow. Nat. Comm. 5, 3820.

Kazerooni, H. T., Fornari, W., Hussong, J. \& BRANDT, L. 2017 Inertial migration in dilute and semidilute suspensions of rigid particles in laminar square duct flow. Phys. Fluids 2, 084301 .

Kidanemariam, A. G., Chan-Braun, C., Doychev, T. \& Uhlmann, M. 2013 Direct numerical simulation of horizontal open channel flow with finite-size, heavy particles at low solid volume fraction. New J. Phys. 15, 025031.

Kulick, J. D., Fessler, J. R. \& EAton, J. K. 1994 Particle response and turbulence modification in fully developed channel flow. J. Fluid Mech. 277, 109-134.

Lashgari, I., Picano, F., Breugem, W.-P. \& Brandt, L. 2014 Laminar, turbulent, and inertial shear-thickening regimes in channel flow of neutrally buoyant particle suspensions. Phys. Rev. Lett. 113, 254502. 
Lathrop, D. P., Fineberg, J. \& Swinney, H. L. 1992 Turbulent flow between concentric rotating cylinders at large Reynolds number. Phys. Rev. Lett. 68, 1515.

LEWIS, G. S. \& SWINNEY, H. L. 1999 Velocity structure functions, scaling, and transitions in high-Reynolds-number Couette-Taylor flow. Phys. Rev. E 59, 5457.

LiU, T. J. \& BANKoff, S. G. 1993 Structure of air-water bubbly flow in a vertical pipe - I. Liquid mean velocity and turbulence measurements. Intl J. Heat Mass Transfer 36 (4), 1049-1060.

Lu, J., Fernández, A. \& Tryggvason, G. 2005 The effect of bubbles on the wall drag in a turbulent channel flow. Phys. Fluids 17, 095102.

Machiconne, N.\& VolK, R. 2016 Lagrangian velocity and acceleration correlations of large inertial particles in a closed turbulent flow. Phys. Fluids 28, 035113.

Maryami, R., Farahat, S., Javad Poor, M. \& Shafiei Mayam, M. H. 2014 Bubbly drag reduction in a vertical Couette-Taylor system with superimposed axial flow. Fluid Dyn. Res. 46 (5), 055504.

Mathai, V., Calzavarini, E., Brons, J., Sun, C. \& Lohse, D. 2016 Microbubbles and microparticles are not faithful tracers of turbulent acceleration. Phys. Rev. Lett. 117, 024501.

Mathai, V., Huisman, S. G., Sun, C., Lohse, D. \& Bourgoin, M. 2018 Enhanced dispersion of big bubbles in turbulence. Available at: arXiv:1801.05461.

Mathai, V., Prakash, V. N., Brons, J., Sun, C. \& Lohse, D. 2015 Wake-driven dynamics of finite-sized buoyant spheres in turbulence. Phys. Rev. Lett. 115, 124501.

Mazzitelli, I. M., Lohse, D. \& Toschi, F. 2003 The effect of microbubbles on developed turbulence. Phys. Fluids 15, L5.

Murai, Y. 2014 Frictional drag reduction by bubble injection. Exp. Fluids 55 (7), 1773.

Muste, M. \& PATEl, V. C. 1997 Velocity profiles for particles and liquid in open-channel flow with suspended sediment. ASCE J. Hydraul. Engng 123 (9), 742-751.

Naso, A. \& Prosperetti, A. 2010 The interaction between a solid particle and a turbulent flow. New J. Phys. 12, 033040.

Ostilla-Mónico, R., Stevens, R. J. A. M., Grossmann, S., Verzicco, R. \& Lohse, D. 2013 Optimal Taylor-Couette flow: direct numerical simulations. J. Fluid Mech. 719, 14-46.

PAN, Y. \& BANERJEE, S. 1996 Numerical simulation of particle interactions with wall turbulence. Phys. Fluids 8, 2733.

Paoletti, M. S. \& LAthrop, D. P. 2011 Angular momentum transport in turbulent flow between independently rotating cylinders. Phys. Rev. Lett. 106, 024501.

Peskin, C. S. 2002 The immersed boundary method. Acta Numerica 11, 479-517.

Picano, F., Breugem, W.-P. \& BRandt, L. 2015 Turbulent channel flow of dense suspensions of neutrally buoyant spheres. J. Fluid Mech. 764, 463-487.

Poelma, C., Westerweel, J. \& Ooms, G. 2007 Particle-fluid interactions in grid-generated turbulence. J. Fluid Mech. 589, 315-351.

Procaccia, I., L'vov, V. S. \& BenzI, R. 2008 Colloquium: theory of drag reduction by polymers in wall-bounded turbulence. Rev. Mod. Phys. 80 (1), 225-247.

Roghair, I., Mercado, J. M., Annaland, M. V. S., Kuipers, H., Sun, C. \& Lohse, D. 2011 Energy spectra and bubble velocity distributions in pseudo-turbulence: numerical simulations versus experiments. Intl J. Multiphase Flow 37 (9), 1093-1098.

Shawkat, M. E., Ching, C. Y. \& Shoukri, M. 2008 Bubble and liquid turbulence characteristics of bubbly flow in a large diameter vertical pipe. Intl J. Multiphase Flow 34 (8), 767-785.

So, S., Morikita, H., Takagi, S. \& Matsumoto, Y. 2002 Laser Doppler velocimetry measurement of turbulent bubbly channel flow. Exp. Fluids 33 (1), 135-142.

Stickel, J. J. \& Powell, R. L. 2005 Fluid mechanics and rheology of dense suspensions. Annu. Rev. Fluid Mech. 37, 129-149.

Tagawa, Y., Roghair, I., Prakash, V. N., van Sint Annaland, M., Kuipers, H., Sun, C. \& Lohse, D. 2013 The clustering morphology of freely rising deformable bubbles. J. Fluid Mech. 721, R2.

Toschi, F. \& Bodenschatz, E. 2009 Lagrangian properties of particles in turbulence. Annu. Rev. Fluid Mech. 41, 375-404. 
Tsuji, Y., Morikawa, Y. \& ShIomi, H. 1984 LDV measurements of an air-solid two-phase flow in a vertical pipe. J. Fluid Mech. 139, 417-434.

Uhlmann, M. 2008 Interface-resolved direct numerical simulation of vertical particulate channel flow in the turbulent regime. Phys. Fluids 20, 053305.

Unverdi, S. O. \& Tryggvason, G. 1992 A front-tracking method for viscous, incompressible, multi-fluid flows. J. Comput. Phys. 100 (1), 25-37.

Verschoof, R. A., VAn der Veen, R. C., Sun, C. \& Lohse, D. 2016 Bubble drag reduction requires large bubbles. Phys. Rev. Lett. 117, 104502.

VReman, A. W. 2015 Turbulence attenuation in particle-laden flow in smooth and rough channels. J. Fluid Mech. 773, 103-136.

Wang, Y., Sierakowski, A. J. \& Prosperetti, A. 2017 Fully-resolved simulation of particulate flows with particles-fluid heat transfer. J. Comput. Phys. 350, 638-656.

White, C. M. \& Mungal, M. G. 2008 Mechanics and prediction of turbulent drag reduction with polymer additives. Annu. Rev. Fluid Mech. 40, 235-256.

Zhao, L. H., Andersson, H. I. \& Gillissen, J. J. J. 2010 Turbulence modulation and drag reduction by spherical particles. Phys. Fluids 22, 081702. 